\section{Virtuelle Leichenschau-Fortbildung}

In Nordrhein-Westfalen können sich Ärzte jetzt via Telelern-Programm zum Thema Leichenschau weiterbilden. Wie die Ärztekammer Westfalen-Lippe (AEKWL) mitteilt, gibt es allerdings zunächst eine Start-Veranstaltung in Präsenzform, in der die rechtlichen und medizinischen Grundlagen der Leichenschau bearbeitet werden. Zudem werde dort die Nutzung der Online-Lernplattform ILIAS der Akademie für Ärztliche Forbtildung der ÄKWL und KVWL sowie der Simulator der Firma INMEDEA GmbH erläutert (www.aekwl.de).

\section{Safe-Net für D- und H-Ärzte}

Durchgangs- und Heilbehandlungsärzte, die via D2D (Doctor to Doctor) am elektronischen Datenaustausch mit den gesetzlichen Unfallversicherungsträgern (DALEUV) teilnehmen, müssen bis Ende des Jahres einen Zugang zum KV-SafeNet haben. Das meldet das Deutsche Gesundheitsnetz (dgn) und beruft sich dabei auf die KV-Telematik-ARGE. Demnach soll die ISDN-Anbindung fürs $\mathrm{D} 2 \mathrm{D}$ für die rund $5.800 \mathrm{D}$ und $\mathrm{H}$-Ärzte nur noch bis Jahresende zur Verfügung stehen.

\section{Neue Forschungsinitiative}

IBM und der Anbieter für Spracherkennung Nuance haben eine Forschungsvereinbarung getroffen. Zweck der Kooperation ist laut einer gemeinsamen Mitteilung die weitere Entwicklung der analytischen Fähigkeiten des IBM Watson-Supercomputersystems für die Gesundheitsbranche. Die Initiative soll die Frage- und Antwort-Methodik, die Verarbeitung der natürlichen Sprache und die Lerneigenschaften des IBM-Systems mit den klinischen Spracherkennungslösungen von Nuance kombinieren. So sollen Ärzte möglicherweise schnelleren Zugang zu wichtigen Informationen erhalten. red

\section{LED-Monitor soll Strom sparen}

Das IT-Unternehmen LG hat einen neuen Monitor vorgestellt, der dank seiner LED-Technologie (LED = Leuchtdiode) 40 $\%$ weniger Strom als herkömmliche LCDMonitore (LCD = Liquid Crystal Display) mit Leuchtstoffröhren-Hintergrundbeleuchtung verbrauchen soll. Der E2290V sei zudem mit seinen 7,2 Millimetern Tiefe der bislang flachste Monitor der Welt.

\title{
Mit Google AdWords zur Visitenkarte der Praxis
}

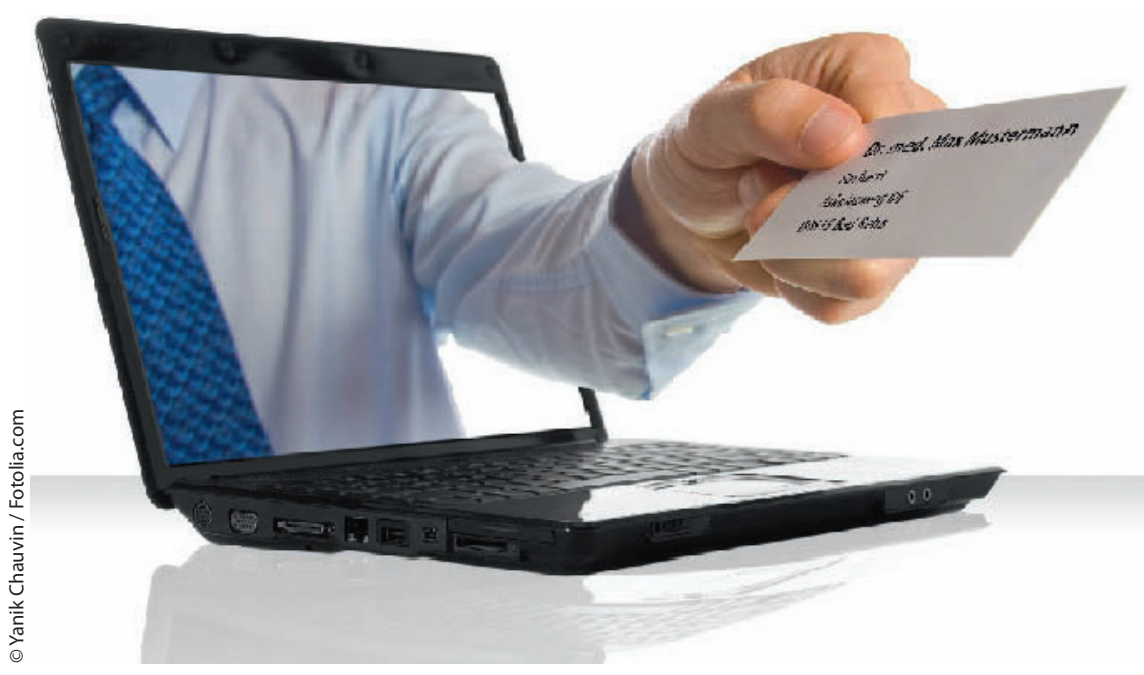

Die Praxis-Website gibt Auskunft über das Leistungsspektrum niedergelassener Ärzte Google AdWords weist Patienten den Weg zur virtuellen Visitenkarte.

Um Patienten anzusprechen und neue Patienten zu gewinnen, führt an der eigenen Praxis-Website kein Weg mehr vorbei. Doch der schönste Internetauftritt bringt wenig, wenn er im World Wide Web untergeht und von potenziellen Interessenten nicht gefunden wird. Eine Lösung für dieses Problem heißt "Google AdWords".

mmerhin $69 \%$ der Deutschen sind mittlerweile zumindest gelegentlich online, so das Ergebnis der ARD/ZDF-Onlinestudie 2010. Kein Wunder, dass auch für Ärzte die eigene Praxis-Website immer wichtiger wird. Damit die Seite auf den Monitoren interessierter Patienten auftauchen, können Ärzte - neben der Listung in Arztbewertungsportalen - Anzeigen im Internet schalten. Dabei seien vor allem die Google AdWords geeignet, erklärt die Online-Marketing-Expertin Sabrina Zebisch von der Convecto New Media GmbH in Offenbach.

Die Funktionsweise ist einfach erklärt: „Sucht ein Nutzer etwa nach 'Hausarzt Frankfurt', bekommt er neben oder über dem Suchergebnis Anzeigen eingeblendet, die sogenannten Google AdWords", sagt Zebisch. Die Vorteile dieser Werbe- form liegen laut Zebisch zum einen darin, dass der Arzt seine Anzeige regional begrenzt schalten kann. So könne ein Arzt nur in Frankfurt, einem oder mehreren Bundesländern oder auch einem selbst definierten Umkreis rund um seine Praxis werben. Die Anzeige wird dann nur Web-Nutzern gezeigt, deren IPAdresse (jedem Rechner im Internet wird eine bestimmte Adresse basierend auf dem Internet Protokoll [IP] - zugewiesen), darauf schließen lässt, dass sie sich in diesem Gebiet aufhalten.

Wichtig für Ärzte ist aber ebenso, dass die Kosten genau kalkulierbar sind. Zebisch: „Der Arzt legt selbst fest, wie viel er monatlich für Google AdWords ausgeben will. Und anders als bei klassischer Bannerwerbung zahlt er nicht für das reine Einblenden seiner Anzeige, son- 